\title{
Percepción de Calidad de Vida en Adultos Mayores Chihuahuenses, Comparaciones por Género
}

\author{
Raúl Barceló Reyna (M. Sc.) \\ Martha Ornelas Contreras (Dra.) \\ Leticia Irene Franco Gallegos (M.A.R.H) \\ Guadalupe Simanga Ivett Robles Hernández (M.C.) \\ Humberto Blanco Vega (Dr.) \\ Edgar Francisco Ordoñez Bencomo (M.C.) \\ Susana Ivonne Aguirre Vásquez (Dra.) \\ Universidad Autónoma de Chihuahua, México
}

\section{Resumen}

La calidad de vida es un estado de satisfacción general que se manifiesta a partir de la percepción de los individuos de su estado físico, psicológico y social. En este orden, el objetivo de esta investigación consistió comparar los perfiles de calidad de vida percibida en adultos mayores chihuahuenses. La muestra total fue de 492 adultos mayores; 291 mujeres y 201 hombres, con edades entre los 60 y 90 años. El abordaje adoptado en la investigación se enmarcó dentro de un enfoque cuantitativo con un diseño descriptivo tipo encuesta. Los resultados del análisis multivariante de la varianza, seguido por los análisis de varianza univariados, muestran que los hombres reportaron una mejor percepción de calidad de vida en los factores función física, vitalidad, rol físico, dolor corporal, rol emocional y salud mental; y sin diferencias significativas en los factores salud general y función social. Estos resultados revelan que en general los hombres en comparación con las mujeres muestran mayores niveles de percepción de calidad de vida. No obstante, es preciso desarrollar más investigación al respecto pues el tema trasciende del todo los alcances de la presente investigación.

Palabras clave: Calidad de vida, género, actitud, autopercepciones 


\title{
Perception of Quality of Life in Elderly Adults from the State of Chihuahua, Gender Comparisons
}

\author{
Raúl Barceló Reyna (M. Sc.) \\ Martha Ornelas Contreras (Dra.) \\ Leticia Irene Franco Gallegos (M.A.R.H) \\ Guadalupe Simanga Ivett Robles Hernández (M.C.) \\ Humberto Blanco Vega (Dr.) \\ Edgar Francisco Ordoñez Bencomo (M.C.) \\ Susana Ivonne Aguirre Vásquez (Dra.) \\ Universidad Autónoma de Chihuahua, México
}

\begin{abstract}
The quality of life is a state of general satisfaction that is manifested from the perception of individuals of their physical, psychological and social status. In this order, the goal of the present research was to compare the profiles of perceived quality of life in elderly participants from the State of Chihuahua. The total sample was 492 elderly people; 291 women and 201 men, with age ranging between 60 and 90 years. The adopted approach was quantitative with a descriptive, survey-like design. Results from the multivariate analyses of variance, followed up by univariate analyses of variance, showed that men report a better perception of quality of life on the factors physical functioning, vitality, physical role, body pain, emotional role and mental health; with no significant differences on the general health and social functioning factors. These results reveal that in general men in comparison to women show higher levels of perception of quality of life. However, it is important to develop more research because the topic trascends the scope of the present study.
\end{abstract}

Keywords: Quality of life, gender, attitude, self perceptions

\section{Introduction}

La sociedad mexicana experimenta un intenso y acelerado cambio en la distribución por edades. En el estado de Chihuahua habitan 3.5 millones de personas, $50.7 \%$ mujeres y $49.3 \%$ son hombres. En la actualidad, la edad media de los chihuahuenses es de unos 28 años, sin embargo, se prevé que para el 2050 se encuentre alrededor de los 39 años. Considerando lo anterior, 
es evidente el aumento del número de personas adultas y adultas mayores, y en un año tan próximo como el 2025 será de un adulto mayor por cada cuatro habitantes, con una expectativa de vida promedio de 80 años aproximadamente (Consejo Nacional de Población, [CONAPO], 2015).

Se consideran adultos mayores aquellas personas que sobrepasan los 60 años o más. A estas, se les denomina también de la tercera edad. Hoy en día dadas las expectativas de vida, se habla de una cuarta edad para aquellas que sobrepasan los 80 años. Un denominador común durante este período es el envejecimiento (CONAPO, 2015).

Desde el punto de vista biológico, el envejecimiento es el resultado de la acumulación de daños moleculares y celulares a lo largo del tiempo. Esto conduce a una disminución progresiva de las capacidades físicas y mentales, así como al incremento del riesgo de padecer enfermedades y finalmente a la muerte (Organización Mundial de la Salud, [OMS], 2018).

Para referirse de un modo apropiado a este proceso, debe tratarse desde una perspectiva integradora, pues el ser humano es un ente biopsicosocial. En este orden, el envejecimiento trae consigo múltiples afectaciones tanto biológicas como psicológicas y sociales. Entre las más frecuentes se destacan la incidencia de las enfermedades crónicas no transmisibles, así como las degenerativas del sistema osteomioarticular, con mayor implicación de los miembros inferiores, propiciando múltiples limitaciones funcionales (Pérez, Barragán, Herrera y Falcón, 2019); (González, 2018); (Barragán, 2016); (Martos, Pozo y Alonso, 2010).

Al mismo tiempo, enfrentan además transiciones que repercuten en la dimensión psicosocial, tales como la jubilación o pérdida de familiares y amigos. Esto, por una parte, limita su participación ciudadana y, por otra, en muchos casos los convierte en dependientes de hijos, otros familiares o instituciones sociales. Lo antes descrito afecta a todos los adultos mayores, sin embargo, presenta como singularidad que lo hace de un modo individual, asincrónico y progresivo.

Los datos expuestos, demandan intervenciones que aporten información valiosa para favorecer el incremento en la calidad de vida de este vulnerable sector poblacional. Esta investigación, se torna más compleja al estudiar la percepción de calidad de vida en la vejez, pues, unido a las particularidades de este período de vida y a la naturaleza multifactorial del constructo, se suman la satisfacción o no de las aspiraciones y necesidades personales (Martínez, Aucapán, Muñoz y Silva, 2018). Esto se corrobora al analizar lo planteado por Flores et al. (2018) al manifestar que, el contexto donde interactúan los adultos mayores determina en gran medida la percepción que reflejen sobre su calidad de vida.

Diversos autores indagaron en relación con la calidad de vida en adultos mayores. Como punto coincidente, refieren que en la medida que 
transcurren los años la calidad de vida se ve afectada, debido a los cambios que ocurren durante el proceso de envejecimiento (Ferreira, Broering, Luza y Piazza, 2015); (Santin, Santos, Cristi y Garatachea, 2017). En este contexto, para poder evaluar con mayor precisión la calidad de vida, es necesaria la percepción de la misma por parte de los sujetos que se investigan. (Bruno, Stutzer, Stephan, Luechinger y Benesch, 2008) y (Haybron, 2013).

Calidad de vida en la vejez, en términos de bienestar general, puede definirse como el estado subjetivo que se le atribuye a la satisfacción con la vida y el equilibrio emocional como reflejo de la solvencia de las necesidades y la capacidad de adaptación de los adultos mayores a las nuevas condiciones de vida (Boggatz, 2016).

La percepción de calidad de vida en la vejez, está condicionada en gran medida por la salud general de los sujetos. A su vez, la autopercepción sobre el estado de salud, está determinado, entre otros aspectos, por la condición física (vista como la resultante de la integración de capacidades y habilidades que le permitan auto valerse para sus actividades de la vida cotidiana) y las enfermedades padecidas (Jodra, Maté y Domínguez, 2019).

Al envejecimiento de la población generalmente lo acompaña una modificación en el perfil epidemiológico, donde se destacan las enfermedades propias de este período unidas al mayor riesgo de caídas con sus posteriores complicaciones. Esta realidad demanda no solo el incremento de los servicios especializados, sino, además una mayor preparación del personal que se encargará de prestarlo (Consejo Estatal de Población, [COESPO], 2017).

Al respecto, para los profesionales de la cultura física que atienden los adultos mayores, el reto fundamental es establecer programas de intervención más efectivos, que potencien una mejor calidad de vida. En este estudio se intenta determinar las diferencias y similitudes entre hombres y mujeres adultos mayores mexicanos en cuanto a su percepción de calidad de vida. En consecuencia, esta investigación pretende, como investigación aplicada, aportar información que se traduzca en una práctica de mayor calidad en el contexto de la atención al adulto mayor.

\section{Método}

\section{Participantes}

Participaron en el estudio 492 adultos mayores, 291 mujeres y 201 hombres de la ciudad de Chihuahua, México, residentes en la comunidad. La edad de los adultos mayores fluctuó entre los 60 y 90 años. El $84 \%$ se encontraban en el rango de 60 a 80 y el $16 \%$ sobrepasan los 80 ; con $\mathrm{M}=71.37$ \pm 8.16 años para las mujeres y $M=71.16 \pm 7.84$ años para los hombres. La muestra se obtuvo mediante un muestreo por conveniencia.

Los criterios de inclusión fueron los siguientes: tener 60 años o más, residir en la ciudad de Chihuahua y participar de manera voluntaria en la 
investigación. Se excluyeron del estudio los sujetos con demencia o enfermedades psiquiátricas graves que imposibilitaran la comprensión del cuestionario. Se atendieron los lineamientos del reglamento de la Ley General de Salud en Materia de Investigación para la Salud de México y se siguió la lista de elementos de consentimiento libre e informado señalado por Mondragón (2009).

\section{Instrumento}

Todos los adultos mayores participantes contestaron el Cuestionario de Salud SF-36 adaptado por Alonso, Prieto y Antó (1995) que consta de 36 ítems, los cuales detectan estados positivos y negativos centrados en el estado funcional y el bienestar emocional; subdivididos en ocho dimensiones: Función física (FF), formada por los ítems 3, 4, 5, 6, 7, 8, 9, 10, 11 y 12; Vitalidad (VT), integrada por los ítems 23, 27, 29 y 31; Rol físico (RF), que agrupa 4 ítems 13, 14,15 y 16; Dolor corporal (DC), compuesto por los reactivos 21 y 22; Salud general (SG),que integra los ítems 1, 33, 34, 35 y 36; Función social (FS), formada por 2 ítems 20 y 32; Rol emocional (RE), compuesta por los ítems 17, 18 y 19; y Salud mental (SM) agrupada en 5 ítems 24, 25, 26, 28 y 30; adicionalmente, el SF-36 incluye una pregunta de transición (ítem 2) sobre el cambio en el estado de salud general con respecto al año anterior. Este ítem no se utiliza para el cálculo de ninguna de las 8 dimensiones principales.

Las opciones de respuestas son opciones tipo Likert, donde el número de opciones varia de dos a seis puntos, dependiendo del ítem; tal es el caso de los reactivos 3 y 6 que son de 3 puntos y miden la función física que van desde 1 es sí, me limita mucho hasta 3 es no, me limita nada; otros reactivos como el 13 y 14 miden el rol físico muestran respuestas dicotómicas: 1 es sí y 2 es no; reactivos como el 22 que mide el dolor corporal, presenta puntuaciones de 1 a 5 puntos, donde 1 es nada y 5 mucho; y finalmente reactivos como 28 y 29 que miden salud mental y vitalidad respectivamente, presentan puntuaciones de 1 a 6 puntos, donde 1 es siempre y 6 es nunca; los reactivos se registran inversamente a fin de que guarden el mismo sentido.

Para nuestro estudio se hicieron tres adaptaciones a la versión de Alonso et al. (1995):

En la versión utilizada en la presente investigación el sujeto, en todos ítems, elige entre 11 posibles respuestas. Conjugamos la escala original con nuestra versión para que quedara de la manera siguiente: nada (0), poco $(1,2$ y 3), regular (4, 5 y 6), mucho (7, 8 y 9) y bastante (10). Esta primera adaptación se llevó a cabo con la intención de obtener una mayor variabilidad en las respuestas. 
La segunda adaptación consistió en cambiar algunos términos utilizados en los ítems de la versión original con el fin de utilizar un lenguaje más adecuado al contexto de la cultura mexicana.

La tercera adaptación consistió en aplicar el instrumento por medio de una computadora (Figura 1); esto con el fin de permitir el almacenamiento de los datos sin etapas previas de codificación, con una mayor precisión y rapidez.

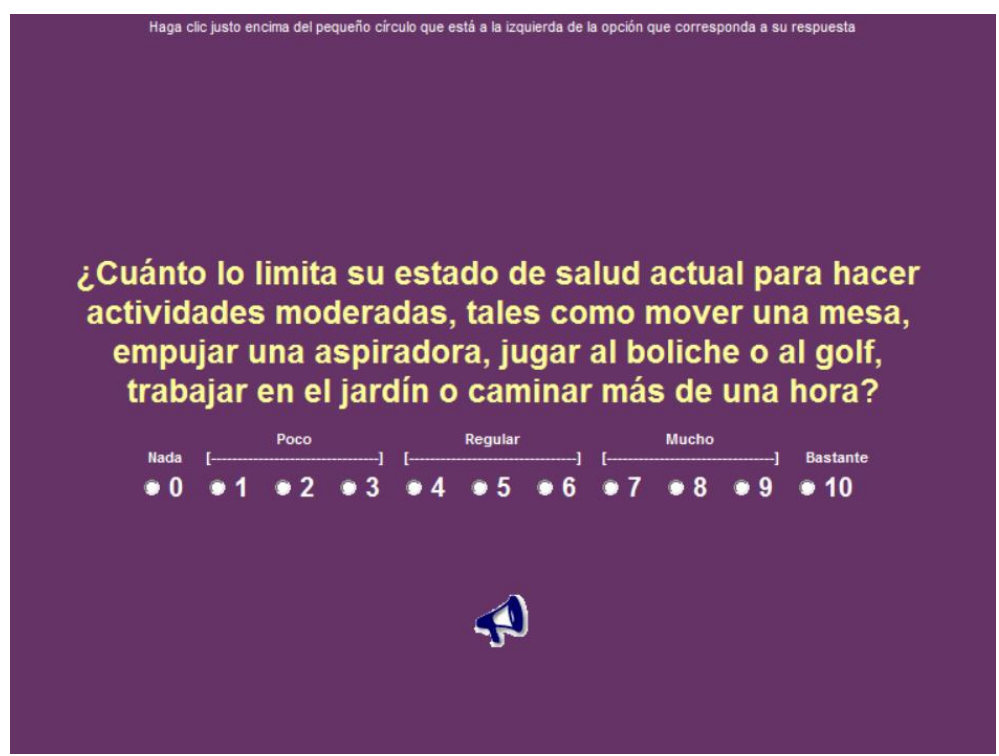

Figura 1. Ejemplo de respuesta para los ítems del cuestionario

\section{Diseño}

En cuanto al diseño del estudio, se utilizó un enfoque cuantitativo con un diseño descriptivo y transversal tipo encuesta (Hernández, Fernández, \& Baptista, 2014). La variable independiente fue el género y las variables dependientes el promedio de las puntuaciones obtenidas en los ocho factores del Cuestionario de Salud SF-36: Función física, Vitalidad, Rol físico, Dolor corporal, Salud general, Función social, Rol emocional y Salud mental.

\section{Procedimiento}

Se invitó a participar en el estudio a adultos mayores de la ciudad de Chihuahua, México. Los que aceptaron participar firmaron la carta de aceptación correspondiente. Luego se aplicó el instrumento, antes descrito, por medio de una computadora personal (módulo administrador del instrumento del editor de escalas de ejecución típica), en una sesión de aproximadamente 45 minutos. Al inicio de cada sesión se hizo una pequeña introducción sobre la importancia de la investigación y de cómo acceder al instrumento. Se les solicitó la máxima sinceridad y se les garantizó la confidencialidad de los 
datos que se obtuvieran. Las instrucciones de cómo responder se encontraban en las primeras pantallas; antes del primer reactivo del instrumento. Al término de la sesión se les agradeció su participación. Una vez aplicado el instrumento se procedió a recopilar los resultados por medio del módulo generador de resultados del editor de escalas versión 2.0 (Blanco et al., 2013).

\section{Análisis de datos}

Se realizaron análisis estadísticos descriptivos (medias y desviaciones estándar) para todas las variables dependientes. Posteriormente, después de verificar que los datos cumplían con los supuestos de los análisis estadísticos paramétricos, se llevó a cabo un análisis multivariante de varianza (MANOVA), seguido por los análisis de varianza univariados (ANOVAs), para examinar las diferencias entre mujeres y hombres en cuanto a su percepción de calidad de vida en los factores Función física, Vitalidad, Rol físico, Dolor corporal, Salud general, Función social, Rol emocional y Salud mental, el tamaño del efecto se estimó mediante el eta cuadrado $\left(\eta^{2}\right)$. Todos los análisis estadísticos se realizaron con el programa SPSS versión 21.0 para Windows. El nivel de significación estadística se estableció en $p=.05$.

\section{Resultados}

La Tabla 1 muestra los valores de las medias y las desviaciones estándar de la percepción de calidad de vida en los ocho factores del Cuestionario de Salud SF-36, así como los resultados del MANOVA y los subsiguientes ANOVAs. Los resultados del MANOVA indicaron diferencias significativas globales de acuerdo a la variable género en las puntuaciones de percepción de calidad de vida (Wilks' $\lambda=.864 ; p<.001 ; \eta^{2}=.136$ ). Posteriormente, los resultados de los ANOVAs mostraron que los hombres reportaron una mejor percepción de calidad de vida que las mujeres en seis de los ocho factores estudiados: función física $(F=38.04, p<.001)$, vitalidad $(F$ $=31.913, p<.001)$, rol físico $(F=10.287, p<.01)$, dolor corporal $(F=19.614$, $p<.001)$, rol emocional $(F=4.294, p<.05)$ y salud mental $(F=6.760, p<$ $.01)$. Y sin diferencia en los factores salud general y función social. 
Tabla 1. Resultados MANOVA y ANOVAs para las diferencias en percepción de calidad de vida de acuerdo al género

\begin{tabular}{lccccc}
\hline & $\begin{array}{c}\text { Mujeres } \\
(n=291)\end{array}$ & $\begin{array}{c}\text { Hombres } \\
(n=201)\end{array}$ & $\mathrm{F}$ & $\mathrm{p}$ & $\eta^{2}$ \\
\hline & & & 9.502 & $<.001$ & .136 \\
Función física & $4.70(2.56)$ & $6.24(2.94)$ & 38.054 & $<.001$ & .072 \\
Vitalidad & $4.81(1.42)$ & $5.54(1.39)$ & 31.913 & $<.001$ & .061 \\
Rol físico & $5.96(2.95)$ & $6.86(3.17)$ & 10.287 & $<.01$ & .021 \\
Dolor corporal & $5.10(2.94)$ & $6.30(2.94)$ & 19.614 & $<.001$ & .038 \\
Salud general & $6.22(2.03)$ & $6.53(2.15)$ & 2.626 & $>.05$ & .005 \\
Función social & $6.91(2.63)$ & $7.35(2.69)$ & 3.268 & $>.05$ & .007 \\
Rol emocional & $6.82(2.71)$ & $7.35(2.84)$ & 4.294 & $<.05$ & .009 \\
Salud mental & $6.75(2.00)$ & $7.24(2.13)$ & 6.760 & $<.01$ & .014 \\
\hline
\end{tabular}

Nota. Los valores descriptivos se presentan como media (desviación estándar)

\section{Conclusiones}

Los resultados muestran que los hombres son quienes manifiestan una mejor percepción de calidad de vida, sobre todo en lo que se refiere a función física, vitalidad, rol físico, dolor corporal, rol emocional y salud mental; esto no concuerda con lo descrito por López et al. (2019) quienes al realizar este análisis no encontraron diferencias significativas. Aunque al analizar las dimensiones que integran el constructo, reportan que la movilidad física, el dolor y la energía se percibe con mayor afectación en las mujeres. Es oportuno destacar que en dicha investigación se determinó la calidad de vida relacionada con la salud utilizando el cuestionario Perfil de Salud de Nottingham.

En cambio, Gallegos et al. (2019) al estudiar la calidad de vida relacionada con la salud en adultos mayores mexicanos, reportan solo diferencias significativas a favor de los hombres para el factor salud mental, esto quizás se debió a la diferencia en cuanto a la escala de valoración utilizada en cada uno de los ítems, entre la presente investigación y la referida anteriormente.

Por su parte, Rodríguez (2017) no ofrece resultados comparativos entre hombres y mujeres. Sin embargo, declara la no existencia de una relación significativa entre el dolor corporal (provocado por enfermedades osteoarticulares y musculoesqueléticas) y la calidad de vida percibida. No obstante, afirma que la salud mental y la vitalidad son influenciadas significativamente por el dolor crónico.

Las diferencias encontradas entre hombres y mujeres con respecto a la percepción de su calidad de vida sugieren, además, que al diseñar cualquier tipo de intervención que tenga como objetivo la mejora de la misma habrá que tomar en cuenta a la variable género; no obstante, es preciso desarrollar más investigación al respecto pues el tema trasciende del todo los alcances de la 
presente investigación. Se subraya además la importancia de realizar un mayor número de investigaciones sobre el tema en nuestro país.

Por último, cuando menos dos limitaciones están presentes en este trabajo. La primera es que los participantes son solo adultos mayores chihuahuenses, lo que supone una amenaza para la posibilidad de generalizar estos resultados. Ampliar la muestra es un área de trabajo de cara al futuro. La segunda limitación proviene del propio instrumento de medición, que se basa en el autoinforme y que por ello puede contener los sesgos que se derivan de la deseabilidad social.

\section{Agradecimientos}

Este estudio es parte de un proyecto financiado por la Secretaría de Educación Pública, Subsecretaría de Educación Superior-Dirección General de Educación Superior Universitaria de México (DE-13 -6,894). Además, el primer autor disfruta de una beca del Consejo Nacional de Ciencia y Tecnología de México (CONACYT).

\section{References:}

1. Alonso, J., Prieto, L., y Antó, J. (1995). La versión española del SF-36 Health Survey (Cuestionario de Salud SF-36): un instrumento para la medida de los resultados clínicos. Medicina clínica, 104(20), 771-776.

2. Barragán, F. (2016). Present-Absent Older people in the family. Elderly Women in the Family: The Ethics of Care. Polish Social Gerontology Journal 2(12), 25-31. Recuperado de https://www.researchgate.net/profile/Artur_Fabis/publication/326011 056_Family_joys_and_worries_in_the_existential_reflections_of_p eople_in_their_late_adulthood_A_qualitative_research_report/links/5 b337d7ea6fdcc8506d197cc/Family-joys-and-worries-in-theexistential-reflections-of-people-in-their-lateadulthood-A-qualitativeresearch-report.pdf\#page $=25$

3. Blanco, H., Ornelas, M., Tristán, J. L., Cocca, A., Mayorga, D., López, J., \& Viciana, J. (2013). Editor for creating and applying computerise surveys. Procedia Social and Behavioral Sciences, 106, 935-940. doi: 10.1016/j.sbspro.2013.12.105

4. Boggatz, T. (2016). Quality of life in old age-a concept analysis. International journal of older people nursing, 11(1), 55-69. doi: 10.1111/opn.12089

5. Bruno, F., Stutzer, M., Stephan, M., Luechinger, S., \& Benesch, C. (2008). Happiness : a revolution in economics. Munich lectures in economics), 256. 
6. Consejo Estatal de Población. (2017). Programa estatal de población 2017-2021. México: Consejo Estatal de Población Recuperado de http://ceg.chihuahua.gob.mx/PED/EJE5/MedianoP/COESPO.PDF.

7. Consejo Nacional de Población. (2015). La situación demográfica de México. Recuperado de

http://coespo.qroo.gob.mx/Descargas/doc/15\%20ENVEJECIMIENT O\%20POBLACIONAL/ENVEJECIMIENTO\%20DEMOGR\%C3\%8 1FICO\%20EN\%20M\%C3\%89XICO.pdf.

8. Ferreira, T., Broering, J., Luza, M., y Piazza, L. (2015). Institucionalização e atividade física em idosos e suas relações com medo de cair e qualidade de vida. Sci. med, 25(2), ID20184-ID20184. doi: 10.15448/1980-6108.2015.1.20184

9. Flores, B. I., Castillo, Y., Ponce, D., Miranda, C., Peralta, E. G., y Durán, T. (2018). Percepción de los adultos mayores acerca de su calidad de vida. Una perspectiva desde el contexto familiar. Revista de Enfermería del Instituto Mexicano del Seguro Social, 26(2), 83-88.

10. Gallegos, K., Honorato, Y., Macías, N., García, C., Flores, Y., \& Salmerón, J. (2019). Preventive health services and physical activity improve health-related quality of life in Mexican older adults. salud pública de méxico, 61(2), 106-115. doi: 10.21149/9400

11. González, V. R. (2018). Educación del adulto mayor para enfrentar limitaciones derivadas del envejecimiento. Transformación, 14(1), 7080.

12. Haybron, D. M. (2013). Well-Being for Public Policy by Diener, Ed; Lucas, Richard; Schimmack, Uli; Helliwell, John. Ethics, 124(1), 218227. doi: 10.1086/671411

13. Hernández, R., Fernández, C., y Baptista, P. (2014). Metodología de la investigación. México: McGraw- Hill.

14. Jodra, P., Maté, J. L., y Domínguez, R. (2019). Percepción de salud, autoestima y autoconcepto físico en persones mayores en función de su actividad física. Revista de psicología del deporte, 28(2), 01270134.

15. López, F. J., Morales, A., Ugarte, A., Rodríguez, L. E., Hernández, J. L., y Sauza, L. C. (2019). Comparación de la percepción de calidad de vida relacionado con la salud en hombres y mujeres adultos mayores. Enfermería Global, 18(2), 410-425.

16. Martos, M., Pozo, C., y Alonso, E. (2010). Enfermedades crónicas y adherencia terapéutica: relevancia del apoyo social. Almería: Universidad de Almería.

17. Martínez, M. C., Aucapán, A. M., Muñoz, L. C., y Silva, C. N. (2018). Percepciones psicosociales de adultos mayores participantes del programa "más adultos mayores autovalentes" del cesfam "dr. Pedro 
jáuregui”, osorno, Chile. Revista Horizonte de Enfermería, 29(2), 7588. doi: 107764/Horiz_Enferm.29.2.138-151

18. Mondragón Barrios, L. (2009). Consentimiento informado: una praxis dialógica para la investigación. Revista de Investigación Clínica, 61(1), 73-82.

19. Organización Mundial de la Salud. (2018). Envejecimiento y salud. Recuperado de https://www.who.int/es/news-room/factsheets/detail/envejecimiento-y-salud.

20. Pérez, D., Barragán, F., Herrera, J. M., y Falcón, S. (2019). Evaluación del programa de salud y la mejora de la calidad de la atención: un estudio de caso del programa de ortogeriatría. Revista EURASIA de Educación en Matemáticas, Ciencia y Tecnología, 15(11), 1-13.

21. Rodríguez, S. E. (2017). Percepción de la calidad de vida en anciano con dolor crónico secundario a patología osteoarticular y musculoesquelética. Gerokomos, 28(4), 168-172.

22. Santin, F., Santos, A., Cristi, C., \& Garatachea, N. (2017). Effect of 8 months of whole-body vibration training on quality of life in elderly women. Research in Sports Medicine, 25(1), 101-107. doi: 10.1080/15438627.2016.1258638 